\title{
Editorial
}

\section{Nitric Oxide, from its Chemistry to Biology and Disease}

\author{
Qingping Feng*,1 and Jeremy Scott*,2
}

\author{
${ }^{I}$ Department of Physiology and Pharmacology, University of Western Ontario, London, Ontario, Canada; ${ }^{2}$ Divisions of \\ Occupational and Respiratory Medicine, Department and Faculty of Medicine, University of Toronto, Toronto, Ontario, \\ Canada
}

Since the discovery of nitric oxide (NO) about 30 years ago, the field of NO research has been rapidly expanding and new aspects of NO chemistry and biology continue to emerge. Originally identified as a vasodilator, NO is now known to affect many biological processes from bacteria and single-celled eukaryotes to animals and humans. To share the latest development and to promote research on NO, the $6^{\text {th }}$ Canadian Nitric Oxide Society Conference was held in the beautiful campus of the University of Western Ontario, London, Ontario, Canada on June 10-11, 2010. A total of 50 people (principal investigators and their trainees) from ten universities came to the University of Western Ontario to present their latest work on NO. The research presented at this conference covered areas ranging from NO chemistry, biosynthesis, signal transduction, to the physiology and pharmacology of NO. This supplementary issue of The Open Nitric Oxide Journal publishes a selection of review articles on NO and gives a snap shot of the work presented at this conference.

Nitric oxide synthase (NOS) is evolutionarily conserved and its origin can be traced back to bacteria, which have existed for more than three billion years. In the review by Rafferty [1], the structural similarities and differences between animal and bacterial NOS and how such differences may influence the extent and duration of bacterial NO production are examined. The roles of $\mathrm{NO}$ in microorganisms, which include protection against oxidative stress, UV damage and transcriptional regulation, are also discussed. An exciting development is the discovery of a NOS homologue in Naegleria gruberi, a single-celled, freeliving eukaryote. Further characterization of this NOS homologue may provide an important evolutionary link between bacteria and mammalian NOS.

Spratt et al. review studies in the Guillemette laboratory directed at deciphering the exact regulatory elements within calmodulin $(\mathrm{CaM})$ that are directly involved in regulating the

\footnotetext{
*Address correspondence to these authors at the Department of Physiology and Pharmacology, University of Western Ontario, London, Ontario, Canada, E-mail: qfeng@uwo.ca

Divisions of Occupational and Respiratory Medicine, Department and Faculty of Medicine, University of Toronto, Toronto, Ontario, Canada;

Tel: 519-850-2989; E-mail: jeremy.scott@utoronto.ca
}

NOS enzymes [2]. Through the use of various CaM mutants, they have mapped the sites in CaM responsible for the binding and activating the NOS enzymes using a variety of biophysical techniques including kinetic analyses and fluorescence. Their innovative work on CaM-iNOS interactions has led them to propose a novel working model for the CaM-dependent regulation of iNOS. Their studies have improved the present understanding of the CaMdependent binding and activation of the NOS isozymes, particularly the interactions between CaM and iNOS.

An important effect of $\mathrm{NO}$ is the post-translational modification of proteins through $S$-modification, which includes $S$-nitrosylation and $S$-glutathionylation. Identification of sites of $S$-modification in proteins is imperative in the understanding of the location, stability and frequency of these modifications. To this end, Faccenda and Mutus review the current methodologies available for identifying sites susceptible to $S$-modification and histochemical visualization of cellular compartments susceptible to $S$-modification [3]. The advantage and potential pitfalls of each method are outlined. In addition, factors that affect $S$-modification susceptibility are also discussed.

The major function of the red blood cell (RBC) is to supply oxygen to demanding tissues and organs. To achieve this, it is now clear that the RBC itself also regulates blood flow and increases the number of oxygen-carrying cells to the hypoxic tissue. However, the mechanisms by which the $\mathrm{RBC}$ facilitates blood flow are not completely understood. Halpin et al. review information pertaining to the role of the $\mathrm{RBC}$ as both a deliverer of NO, and a stimulator of NO release via generation of ATP [4]. In addition, an evaluation of the potential downstream effects of RBC-derived ATP, via its ability to stimulate $\mathrm{NO}$ in other cell types, is provided. While advances have been made with respect to RBCs and $\mathrm{NO}$, a complete understanding of their roles in vivo is lacking and continued efforts in this area are needed.

L-Arginine is the substrate both NOS and arginase for the production of NO and urea, respectively. Thus, competition for substrate L-arginine between these enzymes may limit NO production, which may have implications in the airways hyper-responsiveness of asthma. A delicate balance between NOS and arginase pathways is critical in maintaining 
bronchomotor tone and homoeostasis in the lung. In the review by North and Scott [5], the reciprocal regulation of the NOS and arginase pathways, and the endogenous NOS inhibitors, methylarginines, and their roles in the airways hyper responsiveness of asthma are discussed. Understanding the role of methylarginines and other Larginine metabolites in diseases of the airways, such as asthma and cystic fibrosis [6], may have therapeutic implications.

Finally, Burger and Feng review current understanding and their own work on the protective role of NO in cardiac arrhythmia [7]. NO donors, nitroglycerin and sodium nitroprusside have long been known to protect the heart from ventricular arrhythmias $[8,9]$. However, these effects were traditionally attributed to the drug's capacity to reduce ischemic damage to the heart, rather than direct actions by NO on cardiac electrophysiology. Interestingly, NOS inhibitors increase arrhythmia, or attenuate anti-arrhythmic actions in a variety of animal models. Additionally, genetic studies from animals deficient in NOS enzymes have found increased susceptibility to pharmacological or ischemiainduced arrhythmia while transgenic mice overexpressing certain NOS isozymes may be protected. The precise mechanisms of NO-mediated protection from arrhythmia are still under investigation but may include reductions in calcium overload, regulation of gap junction/connexin expression, reductions in oxidative stress, and regulation of sympathetic activity [7]. Further studies are required to determine the efficacy of specific NO donors and their effective doses before they can be tested in clinical trials in patients with cardiac arrhythmia.

Collectively, these reviews summarize the recent development and the investigators' own work in a wide area of NO research. Over the last 30 years, NO has been a subject of intense research, and major advances have been made in our understanding on the evolutionarily conserved NOS enzymes. However, there are many unanswered questions on the role of NO in numerous biological functions from bacteria to animals and humans. These reviews may lead to new insights in the understanding of some of these questions, and the annual Canadian Nitric Oxide Society Conference will serve as an excellent forum to exchange ideas and to facilitate NO research.

\section{REFERENCES}

[1] Rafferty, S. Nitric oxide synthases of bacteria and other unicellular organisms. Open Nitric Oxide J., 2011, 3, 25-32.

[2] Spratt, D.E.; Duangkham, Y.; Taiakina, V.; Guillemette, J.G. Mapping the binding and calmodulin-dependent activation of nitric oxide synthase isozymes. Open Nitric Oxide J., 2011, 3, 16-24.

[3] Faccenda, A.; Mutus, B. Current methods for the identification of sites of S-glutathionylation and S-nitrosylation in proteins. Open Nitric Oxide J., 2011, 3, 33-37.

[4] Halpin, S.T.; Anderson, K.; Vogel, P.; Spence, D.M. The red blood cell and nitric oxide: derived, stimulated, or both? Open Nitric Oxide J., 2011, 3, 8-15.

[5] North, M.L.; Scott, J.A. L-Arginine metabolism in the lung: Reciprocal regulation of the NOS and arginase pathways. Open Nitric Oxide J., 2011, 3, 48-54.

[6] Grasemann, H.; Al-Saleh, S.; Scott, J.A.; Shehnaz, D.; Mehl, A.; Amin, R.; Rafii, M.; Pencharz, P.; Belik, J.; Ratjen, F. Asymmetric dimethylarginine contributes to airway nitric oxide deficiency in patients with cystic fibrosis. Am. J. Respir. Crit. Care Med., 2011, 183, 1363-1368.

[7] Burger, D.E.; Feng, Q. Protective role of nitric oxide against cardiac arrhythmia - an update. Open Nitric Oxide J., 2011, 3, 3847.

[8] Kent, K.M.; Smith, E.R.; Redwood, D.R.; Epstein, S.E. Beneficial electrophysiologic effects of nitroglycerin during acute myocardial infarction. Am. J. Cardiol., 1974, 33, 513-516.

[9] Mukherjee, D.; Feldman, M.S.; Helfant, R.H. Nitroprusside therapy. Treatment of hypertensive patients with recurrent resting chest pain, ST-segment elevation, and ventricular arrhythmias. JAMA, 1976, 235, 2406-2409.

(C) Feng and Scott; Licensee Bentham Open.

This is an open access article licensed under the terms of the Creative Commons Attribution Non-Commercial License (http://creativecommons.org/licenses/by-nc/3.0/) which permits unrestricted, non-commercial use, distribution and reproduction in any medium, provided the work is properly cited. 